\title{
Dopamine transporter DAT and receptor DRD2 variants affect risk of lethal cocaine abuse: a gene-gene-environment interaction
}

\author{
D Sullivan ${ }^{1,2}$, JK Pinsonneault ${ }^{1}$, AC Papp ${ }^{1}$, H Zhu², S Lemeshow ${ }^{2}$, DC Mash ${ }^{3}$ and W Sadee ${ }^{1,2}$
}

Epistatic gene-gene interactions could contribute to the heritability of complex multigenic disorders, but few examples have been reported. Here, we focus on the role of aberrant dopaminergic signaling, involving the dopamine transporter DAT, a cocaine target, and the dopamine D2 receptor, which physically interacts with DAT. Splicing polymorphism rs2283265 of DRD2, encoding D2 receptors, were shown to confer risk of cocaine overdose/death (odds ratio $\sim 3$ ) in subjects and controls from the Miami Dade County Brain Bank. ${ }^{1}$ Risk of cocaine-related death attributable to the minor allele of rs2283265 was significantly enhanced to $\mathrm{OR}=7.5(P=0.0008)$ in homozygous carriers of the main 6-repeat allele of $D A T \mathrm{rs} 3836790$, a regulatory VNTR in intron8 lacking significant effect itself. In contrast, carriers of the minor 5-repeat $D A T$ allele showed no significant risk $(O R=1.1, P=0.84)$. DAT rs3836790 and DRD2 rs2283265 also interacted by modulating DAT protein activity in the ventral putamen of cocaine abusers. In high-linkage disequilibrium with the VNTR, DAT rs6347 in exon9 yielded similar results. Assessing the impact of DAT alone, a rare DAT haplotype formed by the minor alleles of rs 3836790 and rs27072, a regulatory DAT variant in the $3^{\prime}$-UTR, occurred in nearly one-third of the cocaine abusers but was absent in African American controls, apparently conferring strong risk. These results demonstrate gene-gene-drug interaction affecting risk of fatal cocaine intoxication.

Translational Psychiatry (2013) 3, e222; doi:10.1038/tp.2012.146; published online 22 January 2013

\section{Introduction}

Genetic factors contribute to complex human traits and diseases, but the extent of genetic influence often remains uncertain. ${ }^{2}$ Addressing the 'mystery of missing heritability', Zuk et al. ${ }^{3}$ posit that interactions among loci (epistasis) may account at least in part for the gap between known genetic risk factors and estimated overall heritability in a population. Gene-gene and gene-environment interactions likely have a critical role, but few studies have demonstrated interacting genes that had a strong impacted on a complex trait, ${ }^{4-8}$ or how these interactions might depend on external conditions ${ }^{9,10}$ ). In C. elegans, genetic variation in catechol/peptide receptors and environmental cues (npr-1/tyra-3/nutrient supply) converge to regulate behavioral decisions, ${ }^{11}$ suggesting an ancient evolutionary role of catecholamine signaling - a rare example of a gene-gene-environment interaction. In humans, several genes involved in behavioral modulation carry frequent variants, often regulating gene expression and mRNA processing or translation. ${ }^{12,13}$ As these variants may be considered to reside within a normal range, they are typically not recognized as disease risk factors in GWAS analyses, but in the context of environmental challenge and epistatic interactions, may incur strong risk. Here, we tested a gene-gene-drug (environment) interaction affecting risk of cocaine-induced death, using statistical genetics methods.

Dopaminergic signaling proteins are thought to contribute strongly to drug addiction. ${ }^{14,15}$ The dopamine transporter DAT (encoded by SLC6A3) regulates neurotransmission by terminating dopamine signaling at the synapse through high affinity reuptake of dopamine into presynaptic terminals. Cocaine and similar stimulant drugs inhibit dopamine reuptake by binding to and blocking DAT, ${ }^{16}$ elevating synaptic dopamine levels-contributing to the 'high' experienced upon cocaine ingestion. Similarly critical in dopaminergic signaling, the dopamine D2 receptor (encoded by DRD2) ${ }^{17}$ occurs in two main splice variants, a short form lacking exon6 (D2S) and a long form including exon6 (D2L). ${ }^{18,19}$ Unlike D2L, D2S is largely expressed presynaptically and is considered an autoreceptor that inhibits dopamine release upon activation. Importantly, D2S and DAT proteins physically interact with each other, with D2S facilitating DAT localization to the presynaptic membrane, augmenting dopamine reuptake. ${ }^{20-23}$

$D A T$ and DRD2 are obvious candidate genes associated with cognitive processes and mental disorders, including drug addiction, ${ }^{1,4,24-34}$ but statistically significant associations often lack evidence for causative relationships. We have identified a $D R D 2$ promoter SNP associated with enhanced expression (rs12364283) and two intronic DRD2 SNPs bracketing exon6, rs2283265 and rs 1076560 , which reduce D2S formation. ${ }^{33}$ Occurring in high linkage disequilibrium (LD) (minor allele frequency (MAF) 18\% in Caucasians), the two intronic SNPs were significantly associated with cognitive processing ${ }^{33}$ and risk of death in heavy cocaine abusers, with

\footnotetext{
${ }^{1}$ Department of Pharmacology and Program in Pharmacogenomics, The Ohio State University, Columbus, OH, USA; ${ }^{2}$ Department of Biostatistics, College of Public Health, The Ohio State University, Columbus, OH, USA and ${ }^{3}$ University of Miami School of Medicine, Departments of Neurology and Molecular and Cellular Pharmacology, Miami, FL, USA

Correspondence: Dr W Sadee, Department of Pharmacology, Program in Pharmacogenomics, The Ohio State University, 333W. 10th Avenue, Columbus, $\mathrm{OH}$ 43210-1239, USA.

E-mail wolfgang.sadee@osumc.edu

Keywords: cocaine-related death; $D A T$; DRD2; epistasis; gene-gene interaction; gene regulation; haplotype

Received 24 October 2012; accepted 10 November 2012
} 
an odds ratio (OR) of $\sim 3 .{ }^{1}$ Risk was not significantly elevated in African American subjects, suggesting the presence of epistasis involving other genes. Similarly, DAT harbors numerous polymorphisms, but evidence for true in vivo functionality was limited. A frequent intron8 5/6-repeat (rs3836790) inserted into reporter gene constructs was found to affect expression in vitro, which is further modulated by cellular signaling including cocaine stimulation, ${ }^{29,30}$ with the 6repeat allele identified as a risk factor in cocaine abuse. ${ }^{29} \mathrm{We}$ have shown that the intron8 5-repeat is associated with reduced DAT mRNA levels in human substantia nigra; however, exon9 rs6347 is in high LD with the 5/6-repeat, and therefore, cannot be excluded as a contributor to altered expression. ${ }^{24}$ Moreover, the $3^{\prime}$-UTR SNP rs27072 (MAF $\sim 18 \%$ ) affects DAT mRNA expression and translation, the latter regulated by the overall DAT mRNA level-a mechanism that may involve microRNA regulation and translational control. ${ }^{24}$ No evidence was obtained for a regulatory role of the $3^{\prime}$-UTR $9 / 10$ repeat (rs28363170), ${ }^{24}$ frequently used in clinical association studies with variable results. ${ }^{28,32}$ In an analysis of both $D A T$ and $D R D 2$ polymorphisms, Kazantseva et $a I^{34}$ found that each was associated with personality traits, but their study was not guided by molecular genetic evidence in the selection of variants and failed to address gene-gene interactions.

Given the direct physical interaction between D2S and DAT protein, and the presence of frequent validated regulatory variants in each locus, we asked whether a gene-gene interaction affects risk of cocaine-induced death, and in comparison, DAT protein expression. Heavy cocaine exposure was shown to cause a substantial upregulation of DAT protein and function measured in human brain autopsies, ${ }^{35} \mathrm{a}$ process likely affected by regulatory $D R D 2$ and $D A T$ variants. Our results reveal a strong gene-gene-environment interaction, affecting cocaine-induced death.

\section{Materials and methods}

Post-mortem human brain tissues. Prefrontal cortex (PFC, Brodmann's area 46) and ventral putamen tissues from cocaine abusers who died from cocaine intoxication and age-matched drug-free controls were provided from an archived biorepository (University of Miami, Miami, FL, USA), as described. ${ }^{1}$ Cocaine abusers with cocaine-related deaths were subjects of medicolegal investigations conducted by forensic pathologists, with cocaine levels, circumstances of death, and other toxicological data available. $^{36}$ The samples consisted of 126 cases (cocaine intoxication deaths) and 99 control subjects (non-drug related death). The average age was 36 (s.d. 11) for the controls, 37 (s.d. 10) for the cases, with $86 \%$ males in each group. Overall, there were 143 whites (Caucasians and Hispanics), 81 African Americans and 1 Pacific Islander.

Brain and blood toxicology were positive for cocaine and metabolites for each case, and most of the drug-exposure cases had several surrogate measures of chronic cocaine abuse (drug-related pathology, arrest records, hospital and treatment admissions). Drug-free age-matched control subjects were selected from accidental or cardiac sudden deaths with negative urine screens for all common drugs, with no history of psychiatric disorders or licit or illicit drug use prior to death.

Genotyping methods. DNA was extracted from all tissues as described. ${ }^{33}$ Three DRD2 and four DAT variants (SNPs and repeat polymorphisms) (Table 1) were genotyped using several methods: rs27072 and rs6347 were genotyped by SNaPshot (Life Technologies, Carlsbad, CA, USA), rs12364283 was genotyped with allele-specific PCR, while rs2283265 and rs1076560 were genotyped via modified PCR-restriction fragment length polymorphism (RFLP) methods. ${ }^{1,24,33}$ Repeat polymorphisms in DAT were PCR amplified with fluorescently labeled primer pairs, followed by analysis on an $\mathrm{ABI} 3730$ sequencer (Life Technologies). ${ }^{24}$ The location of the polymorphisms in DRD2 and DAT is shown in Supplementary Figure 1.

\section{Statistical methods}

Case control analysis. Logistic regression was used to analyze the association between cocaine-induced death and seven functional variants. The initial interest was to study the effect of each variant individually, with the ultimate goal of modeling gene-gene interactions. Single variant models were fit using three genetic models (additive, recessive and dominant), and the likelihood ratio test (LRT) was used to test significance. Age, gender and race were examined for confounding influence, using the criterion of a change in log-OR $>15 \%$, for any variant significant at level 0.20 . Interactions with race were considered for all variants and were tested using significance level $0.02 .{ }^{37}$ Candidates for two-variant interaction models were those significant at level 0.20; a conservative cutoff selected as we hypothesized that variants with only marginally significant effects

Table 1 Polymorphisms of DRD2 and DAT genotyped in this study

\begin{tabular}{lllll}
\hline Marker & Gene & Location & Function & MAF-All subjects \\
\hline rs1076560 $(\mathrm{G}>\mathrm{T})$ & DRD2 & Intron6 & Splicing & 14 \\
rs2283265 $(\mathrm{G}>\mathrm{T})$ & DRD2 & Intron5 & Splicing & 212 \\
rs12364283 $(\mathrm{A}>\mathrm{G})$ & DRD2 & Promoter Region & Transcription & 14 \\
rs3836790 $(6>5)$ & DAT & Intron8 5/6 Repeat & Regulatory & 5.8 \\
rs6347 $(\mathrm{A}>\mathrm{G})$ & DAT & Exon9 & Potentially regulatory & 39 \\
rs27072 $(\mathrm{C}>\mathrm{T})$ & DAT & 3'UTR (Exon15) & Regulatory & 39 \\
rs28363170 $(10>9)$ & DAT & 3'UTR 9/10 Repeat & Uncertain evidence & 223 \\
& & & 224
\end{tabular}

For DRD2 evidence describing the functions of each variant see Moyer et al. and Zhang et al. ${ }^{1,33}$ For DAT evidence see Pinsonneault et al. ${ }^{24}$ Genomic location of these variants is shown in Supplementary Figure 1. 
themselves may still have the capacity to exert strong epistatic interactions. This approach follows conventional model building strategies. Each candidate variant was tested for interaction with all variants using a more-stringent final significance level of 0.02 . Dominant models were used to accommodate less frequent variants. The role of race was also examined in each final interaction model. However, the number of independent genetic variants that entered the final analysis was rather small, as DAT rs6347 and intron8 rs38366790 are in high LD, as are DRD2 rs2283265 and rs 1076560, whereas DRD2 rs12364283 promoter SNP was too infrequent to yield robust results. Having intermediate allele frequency, DAT rs27072 was considered independently, particularly in the context of DAT haplotypes, whereas our previous results had failed to assign a regulatory function to the 9/10 repeat rs28363170; for comparison to earlier studies, the 9/10 repeat was included here as well.

Measured genotypes were lacking in a few samples (Table 2), assumed missing completely at random, and available-case analysis was implemented to reflect all available data. ${ }^{38}$ STATA 11 (StataCorp LP, College Station, TX, USA) was used for statistical analyses.

Haplotype analysis. Haplotype frequencies of DAT variants (rs6347, intron8 rs38366790, rs27072, 9/10 repeat rs2836317) were estimated using the EM algorithm separately by race, and case status, with both STATA's haplologit command and R's haplo.em (haplo.stats package from R software; http://cran.r-project.org/). R's haplo.stats package served to estimate posterior probabilities of diplotypes. STATA's haplologit command ${ }^{39}$ was used to model the association between haplotypes and case status, accounting for variability in haplotype frequency estimates and using the appropriate likelihood when the covariates are measured with error. ${ }^{39}$

Analysis of DRD2 and DAT variant effects on DAT Bmax activity. Ventral putamen tissue was employed for the following assays as this brain region has been shown to have a high density of DAT protein. ${ }^{40}$ Striatal DAT Bmax (pmol g ${ }^{-1}$ tissue wet weight) levels were previously obtained from Scatchard plots using $\left[{ }^{3} \mathrm{H}\right]$ carboxyfluorotropane (WIN $35428)^{35,41}$ in ventral putamen tissues from 65 subjects of the 225 total cases and controls. Estimated $K_{d}$ values did not differ significantly across individuals and treatment, and therefore were not included with the analysis. Of the 32 cases and 33 controls, 28 males were in each. Caucasians accounted for 20 cases and 25 controls. Linear regression models served to analyze predictors of $B_{\max }\left(\mathrm{pmolg}^{-1}\right)$, to test two-variant effects on $B_{\max }\left(\mathrm{pmolg}^{-1}\right)$ levels as done with case-control status. Effects may exist in cases but not in controls, and thus models were built separately within each. Significance of variables was assessed by LRT $P$-values. Model assumptions were checked via normal quantile plots and plotting the residuals versus fitted values.

\section{Results}

Analysis of DRD2 and DAT variants. We genotyped three DRD2 and four DAT variants (Table 1; Supplementary Figure 1) in the Miami cohort of 126 cases (deaths due to cocaine intoxication) and 99 controls, as described in Moyer et al.," but employing a slightly larger number of subjects. Allele frequencies are provided in Supplementary Table 1, and LD values are in Supplementary Table 2, showing substantial differences between Caucasians and African Americans. DRD2 splicing variant rs2283265 (and rs1076560 having nearly identical frequencies) and promoter SNP rs12364283 were less frequent in African Americans (7.0 versus $18 \%$ and 1.4 versus $8.2 \%$, respectively). In contrast, the 5 -repeat of $D A T$ rs3836790 was more prevalent in African Americans (66 versus 25\%). Being in LD with rs3836790, DAT rs6347 has similar distributions (Supplementary Table 2). The $3^{\prime} U T R$ variant rs27072 was nearly equal in frequency in Caucasians and African Americans (MAF 15 and 17\%, respectively), while the 9-repeat of rs28363170 was more frequent in Caucasians than African Americans (30 versus 22\%).

Table 2 Main effect of $D A T$ and $D R D 2$ variants on the odds of cocaine-induced death

\begin{tabular}{|c|c|c|c|c|c|c|c|c|c|c|}
\hline \multirow[b]{2}{*}{ GM } & \multirow[b]{2}{*}{ Variant } & \multicolumn{3}{|c|}{ All subjects } & \multicolumn{3}{|c|}{ Caucasians } & \multicolumn{3}{|c|}{ African Americans } \\
\hline & & $\mathbf{N}$ & OR (95\% CI) & P-value & $N$ & OR $(95 \% C l)$ & P-value & $N$ & OR $95 \% C I$ & P-value \\
\hline $\mathrm{D}$ & rs1076560 & 212 & $2.0(1.0,3.8)$ & 0.04 & 137 & $3.3(1.5,7.3)$ & 0.002 & 74 & $0.5(0.1,1.9)$ & 0.29 \\
\hline$A$ & & 212 & $1.8(1.0,3.1)$ & 0.03 & 137 & $2.5(1.3,4.8)$ & 0.004 & 74 & $0.7(0.2,2.2)$ & 0.50 \\
\hline D & rs2283265 & 222 & $2.3(1.2,4.5)$ & 0.01 & 142 & $4.1(1.8,9.2)$ & 0.0003 & 79 & $0.6(0.1,2.2)$ & 0.41 \\
\hline$A$ & & 222 & $2.2(1.2,3.9)$ & 0.005 & 142 & $3.3(1.6,6.8)$ & 0.0002 & 79 & $0.7(0.2,2.4)$ & 0.62 \\
\hline D & rs12364283 & 206 & $1.4(0.6,3.4)$ & 0.49 & 134 & $1.3(0.5,3.4)$ & 0.59 & 69 & - & - \\
\hline$A$ & & 206 & $1.3(0.6,2.8)$ & 0.58 & 134 & $1.2(0.5,2.8)$ & 0.67 & 69 & - & - \\
\hline D & rs3836790 & 223 & $1.4(0.8,2.4)$ & 0.22 & 143 & $1.1(0.6,2.1)$ & 0.84 & 79 & $2.4(0.5,11.4)$ & 0.28 \\
\hline$A$ & & 223 & $1.4(1.0,2.0)$ & 0.08 & 143 & $1.1(0.6,1.8)$ & 0.83 & 79 & $1.9(0.9,3.9)$ & 0.10 \\
\hline D & rs6347 & 224 & $1.2(0.7,2.0)$ & 0.57 & 143 & $0.9(0.5,1.8)$ & 0.84 & 80 & $1.2(0.4,4.3)$ & 0.75 \\
\hline$A$ & & 224 & $1.2(0.8,1.7)$ & 0.42 & 143 & $0.9(0.6,1.6)$ & 0.80 & 80 & $1.3(0.6,2.5)$ & 0.51 \\
\hline D & rs27072 & 225 & $1.0(0.5,1.8)$ & 0.93 & 143 & $0.6(0.3,1.2)$ & 0.16 & 81 & $2.3(0.8,6.8)$ & 0.10 \\
\hline$A$ & & 225 & $1.1(0.6,1.8)$ & 0.81 & 143 & $0.6(0.3,1.3)$ & 0.19 & 81 & $2.4(0.9,6.3)$ & 0.06 \\
\hline D & rs28363170 & 223 & $0.8(0.5,1.3)$ & 0.39 & 143 & $0.9(0.5,1.8)$ & 0.81 & 79 & $0.6(0.2,1.5)$ & 0.29 \\
\hline$A$ & & 223 & $0.8(0.6,1.3)$ & 0.43 & 143 & $0.9(0.6,1.5)$ & 0.77 & 79 & $0.7(0.3,1.6)$ & 0.40 \\
\hline
\end{tabular}

Each variant was fit under three genetic models (GM): dominant, additive and recessive. The dominant (D) and additive (A) models gave comparable results, and are shown above, whereas the recessive model did not yield significant associations. Results for rs12364283 were not estimable in African Americans. 
Single variant cocaine case-control associations. Logistic regression results were obtained for each variant under dominant, recessive and additive genetic models. For assessment of the less frequent DRD2 rs12364283 promoter variant, the study group is too small for achieving sufficient power even for a large effect size, whereas the recessive model did not yield significant results for any variant, suggesting that each allele contributes to the effect sizes observed. As reported earlier, ${ }^{1}$ both DRD2 rs1076560 and DRD2 rs2283265 $(\mathrm{G}<\mathrm{T})$ are significantly associated with odds of cocaine-induced death (Table 2). With both in high LD ( $r^{2} 0.94$, Supplementary Table 2$)$, we selected only DRD2 rs2283265 for further analysis. DRD2 rs2283265 was significant for both dominant and additive models (crude OR 2.3, 2.2; $P=0.012,0.005$, respectively). Interpretation of the dominant model indicates minor T-allele carriers have 2.3 times the odds of cocaine-induced death than GG carriers. The interaction with race is significant for the dominant model $(P=0.015)$, which estimates an OR of 4.1 for Caucasians ( $P=0.0003)$, but a non-significant OR for African Americans (OR $0.57, P=0.41$ ). The T allele appears to present a large risk only in Caucasians, as reported. ${ }^{1}$ Similar results were obtained with the additive model (Table 2).

None of the $D A T$ variants reached level $P=0.05$ significance (Table 2). The intron8 5-repeat (rs3836790) might incur slight risk according to either a recessive (not shown) or additive model (OR 1.9, 1.3; $P=0.077,0.08$, respectively). Adjusting for race decreases the log-odds by $16 \%$, which removes any indication of an association ( $P=0.2$, additive). However, intron8 5/6-repeat allele frequency is associated with race, with African Americans having a higher frequency of the 5-allele (consistent with HapMap data). To prevent problems with colinearity, and as race is not significantly associated with case-status, it was removed from the model. This will be discussed further in the interaction models.

The DAT variant rs28363170 (9/10-repeat) failed to show significant associations and was not further considered here, consistent with our previous results showing no regulatory effects of rs28363170. ${ }^{24}$

Two-variant interaction models in assessing risk of cocaine-related death. The main candidates selected for two-variant interaction models (univariate level 0.20 significance) are DRD2 rs2283265 and intron8 5/6-repeat (rs3836790). Each was uniquely tested for interacting with all other variants, using dominant genetic models to accommodate less frequent alleles. DRD2 rs2283265 significantly interacted with DAT intron8 5/6 repeat (rs3836790) (Table 3a). The interaction model with DRD2 rs2283265 and $D A T$ intron8 5/6-repeat (rs3836790) yielded a significant improvement over the main-effects-only model (LRT $P=0.007)$ and was significantly better than the naive model $(P=0.0015)$. The OR of cocaine-induced death associated with rs2283265 varies strongly with intron8 genotype (Table $3 \mathrm{~b}$ and Figure 1a). In carriers homozygous for the main intron8 6-repeat allele of DAT, the DRD2 rs2283265 OR is $7.5(P=0.0008$, the minor $\mathrm{T}$ allele being the risk factor), in contrast to only 2.3 when considering DRD2 alone (Table 2). Applied only to Caucasians in whom the risk allele combination occurs considerably more frequent than in African Americans, the OR is $6.7 \quad(P=0.002$; data not shown), compared with 4.1 for Caucasians when only considering DRD2 (Table 2). Conversely, in the presence of the intron8 5-repeat allele, the OR for rs2283265 did not differ significantly from unity (OR 1.1, $P=0.84$; Table $3 b$ ). Therefore, the DAT intron8 6-repeat appears to potentiate risk conveyed by the DRD2 rs2283265 T allele, whereas the 5-repeat was protective (the predominant allele in African Americans).

The effect of DAT intron8 5/6 repeat is illuminated by examining the ORs when accounting for different DRD2 rs2283265 genotypes. Although the intron8 5/6 repeat considered alone does not have a significant OR (Table 2), the 5-repeat allele appears to convey significant risk among

Table 3 Interactions between DRD2 rs2283265 (intron5) and DAT rs3836790 (intron8 5/6-repeat) affecting odds of cocaine-induced death

\begin{tabular}{|c|c|c|c|}
\hline & \multicolumn{2}{|c|}{ Coefficient (95\% CI) } & Wald P-value \\
\hline \multicolumn{4}{|l|}{ (a) } \\
\hline Intercept & \multicolumn{2}{|c|}{$-0.42(-0.90,0.09)$} & 0.109 \\
\hline \multicolumn{4}{|l|}{ Main effects } \\
\hline rs2283265 ((GT,TT) vs GG) & \multirow{2}{*}{\multicolumn{2}{|c|}{$\begin{array}{r}2(0.83,3.2) \\
0.76(0.12,1.4)\end{array}$}} & \multirow{2}{*}{$\begin{array}{l}0.0008 \\
0.019\end{array}$} \\
\hline rs3836790 ((56,55) vs 66) & & & \\
\hline \multicolumn{4}{|l|}{ Interaction } \\
\hline rs2283265*rs3836790 & \multicolumn{2}{|c|}{$-1.9(-3.4,-0.52)$} & 0.01 \\
\hline Fixed genotype & Comparison & OR (95\% Cl) & P-value \\
\hline \multicolumn{4}{|l|}{ (b) } \\
\hline$D A T$ rs3836790 & DRD2 rs2283265 & & \\
\hline 66 & (GT,TT) vs GG & $7.5(2.3,25)$ & 0.0008 \\
\hline 56,55 & $(\mathrm{GT}, \mathrm{TT})$ vs $\mathrm{GG}$ & $1.1(0.46,2.6)$ & 0.84 \\
\hline DRD2 rs2283265 & $D A T$ rs3836790 & & \\
\hline $\mathrm{GG}$ & $(56,55)$ vs 66 & $2.1(1.1,4.0)$ & 0.02 \\
\hline GT,TT & $(56,55)$ vs 66 & $0.3(0.08,1.2)$ & 0.08 \\
\hline
\end{tabular}

(a) The logistic regression model indicates a significant interaction term (Wald $P$-value 0.010; LRT $P$-value 0.007) showing that the effect of $D R D 2$ rs2283265 changes with regard to the genotype of DAT rs3836790, and vice versa.

(b) Calculated odds ratios and corresponding confidence intervals obtained through linear combinations of the regression coefficients, for the interaction between $D A T$ rs3836790 and DRD2 rs2283265. 

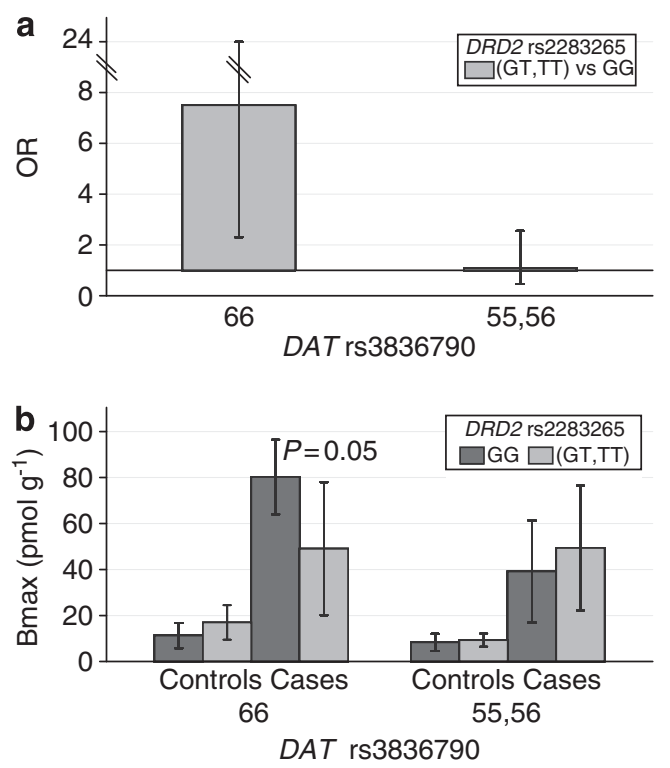

Figure 1 Interaction model between DRD2 rs2283265 and DAT rs3836790 (intron8 5/6-repeat) in the associations with odds of cocaine-induced death (Panel a) and DAT protein $B_{\max }$ activity (Panel b). Panel (a): Interaction model for cocaineinduced death. Odds ratios (ORs) were calculated for the minor vs major alleles of rs2283265 grouped by genotype of DAT rs 3836790 . Error bars are $95 \%$ confidence intervals, as listed in Table 3b. Panel (b): DAT $B_{\max }\left(\mathrm{pmol}^{-1}\right)$ levels. Shown are the levels in cases and controls, grouped by genotype for DRD2 rs228325 and separated by genotypes of DAT rs3836790 (intron8 $5 / 6$ repeat). Error bars are mean $+/-$ s.d. (31 cases and 32 controls for intron8 $5 / 6$ repeat).

homozygous carriers of the major rs2283265 $\mathrm{G}$ allele (OR 2.1, $P=0.02$; Table $3 b$ ), and is possibly protective among carriers of the minor rs2283265 T allele (OR 0.3, $P=0.08$; Table $3 b$ ).

Race was not significantly associated with case status in the DAT intron8 5/6 repeat versus DRD2 rs2283265 interaction model $(P=0.33)$, though it did dampen the effect of intron8, as seen in the univariate models. With race in the model, the interaction $P=0.01$. The OR for $D R D 2$ remained the same $(7.5, P=0.0008)$, but intron8 was no longer as strong a risk factor (OR $1.8, P=0.1$ ). One could argue that only one variable (intron8 or race) is needed in the model. Since the interaction with intron8 is stronger than the interaction with race, intron8 belongs in the model. The interaction with race could be the result of the high frequency of the 5-repeat allele for intron8, and thus intron8 genotype explains why the interaction with race was significant. The race-gene-gene interaction was non-estimable.

Interactions between DRD2 rs2283265 and DAT rs6347 $A>G$ followed a similar pattern, albeit with somewhat lower significance and effect size (Supplementary Table 3). The 3-way race-gene interaction was estimable, though insignificant $(P=0.13)$. When the rs2283265-rs6347 model was fit in only Caucasians, the DRD2 OR was 10.2 among subjects homozygous for the A allele $(P=0.003)$, the one instance where rs6347 had a stronger role. Owing to relatively high LD between DAT rs6347 and intron $85 / 6$-repeat $\left(D^{\prime}=\sim 0.8\right.$, $r^{2}=0.56$, Supplementary Table 2$)$, we cannot ascertain whether rs6347 has independent effects.
Table 4 Two-variant DAT haplotype frequency distributions, by race

\begin{tabular}{|c|c|c|c|c|c|c|}
\hline \multirow[t]{2}{*}{ Haplotype } & \multicolumn{3}{|c|}{ Caucasians } & \multicolumn{3}{|c|}{ African Americans } \\
\hline & $\begin{array}{c}A I I \\
(\mathrm{~N}=143)\end{array}$ & $\begin{array}{c}\text { Cases } \\
(\mathrm{N}=76)\end{array}$ & $\begin{array}{c}\text { Controls } \\
(\mathrm{N}=67)\end{array}$ & $\begin{array}{c}A I I \\
(\mathrm{~N}=81)\end{array}$ & $\begin{array}{c}\text { Cases } \\
(\mathrm{N}=50)\end{array}$ & $\begin{array}{c}\text { Controls } \\
(\mathrm{N}=31)\end{array}$ \\
\hline $11(6 C)$ & 0.63 & 0.63 & 0.63 & 0.32 & 0.27 & 0.33 \\
\hline $12(6 \mathrm{~T})$ & 0.13 & 0.12 & 0.13 & 0.03 & 0.02 & 0.10 \\
\hline $21(5 \mathrm{C})$ & 0.23 & 0.25 & 0.20 & 0.53 & 0.52 & 0.58 \\
\hline 22 (5T) & 0.01 & 0 & 0.04 & 0.12 & 0.19 & 0 \\
\hline
\end{tabular}

Haplotypes of DAT rs3836790 (intron8 5/6-repeat) and DAT rs27072 (3'UTR) were estimated using the EM algorithm, stratified by race, then by case-control status.

Haplotype-associated risk in African Americans. The finding of possible risk of cocaine-related death associated with DAT 3'UTR rs27072 with potentially opposite effects in Caucasians and African Americans (Table 2; but lacking nominal significance) prompted analysis of haplotype structures. Haplotype frequencies estimated from the four DAT variants (rs6347, intron8, rs27072, 9/10 repeat), stratified by case status and race (Supplementary Table 4) demonstrate substantial differences between Caucasians and African Americans, and between cases and controls for haplotype $2221(1=$ major allele, $2=$ minor allele) in African Americans. The 2221 haplotype frequency was estimated to be $16 \%$ among the 50 cases, but $<0.001 \%$ among the 31 controls (Supplementary Table 4).

Further analysis focused on 2-allele DAT haplotypes employing the intron8 5/6-repeat and rs27072 (Table 4). Haplotype frequencies were not significantly associated with case status in Caucasians (haplologit analysis, results not shown). Among African Americans, the haplotype consisting of the intron8 5-repeat and the rs27072 minor allele (5-repeat and T allele, 22-haplotype) reached an estimated frequency of $19 \%$ in cases, with $>1 / 3$ of the cases being carriers, but was $0 \%$ in the controls (Table 4), preventing modeling the effect of the haplotype using haplologit. Eleven subjects had three or more minor alleles resulting in unambiguous phasing with at least one copy of the 22-haplotype, all of whom were cases. Of the 11 subjects with ambiguous phasing (1 minor allele at each variant), 7 were cases and 4 were controls. These results yield a posterior probability for diplotype 11/22 (6C/5T) of 0.80 for the 7 ambiguous cases, but 0 for the controls.

If one ignores phasing of the 2-variant haplotype and only considers the number of minor alleles for each African American, the OR for cocaine related death is 11.6 comparing three or more minor alleles with less than three alleles ( $P=0.006$, exact logistic regression). Comparing instead three or more minor alleles with less than two, we obtain an OR of 2.25 (exact $P=0.086$ ). This result indicates that the intron8 5-repeat and rs27072 T alleles must be in phase on the same haplotype to convey strong risk (22-haplotype).

Effects of DRD2 and DAT variants on DAT protein expression in ventral putamen. Striatal dopamine transporter $B_{\max }$ values were obtained from previous $\left[{ }^{3} \mathrm{H}\right]$ WIN 35428 experiments. ${ }^{35,41}$ DAT $B_{\max }$ levels increased substantially in cocaine abusers $\left(49 \mathrm{pmolg}^{-1}\right.$ tissue in cases versus $10 \mathrm{pmol} \mathrm{g}^{-1}$ in controls; two-sided unequal variance $t$ test, $P=1.5 \mathrm{e}-09$ ) (Figure $1 \mathrm{~b}$, Supplementary Table 6A). 
Owing to the large $B_{\max }$ differences between cocaine abusers and controls, genotype models were formed separately within each subgroup, also coinciding with a prior hypothesis that effects would differ. Among the DRD2 and $D A T$ variants, only DAT rs3836790 (intron8 5/6-repeat) showed association with $B_{\max }$ levels (Supplementary Table 6B). In cases, the estimated coefficient is -24.9 (s.e. 9.6, $P=0.009$ ); in controls, the estimated coefficient is -2.99 (s.e. 1.6, $P=0.07$ ). For this measure, the DAT $9 / 10$ repeat also showed significance, but only in controls $(\beta=3.6$, s.e. 1.6, $P=0.024)$.

Analyzing interactions between $D R D 2$ rs2283265 and DAT rs3836790 or rs6347, separately in cases and controls, no significant interactions were detectable in the controls $(P=0.22$ and 0.51 , respectively). In contrast, significant interactions were observed in the cases for both rs3836790/ rs2283265 (interaction LRT $P=0.028$; overall LRT $P=0.016$; $R^{2} 31 \%$ ) and rs6347/rs2283265 (interaction LRT $P=0.019$; overall LRT $P=0.088, R^{2} 20 \%$ ). Interaction estimates indicate that among cases lacking the 5-repeat allele at intron8, those with the minor $T$ allele at rs2283265 have $31 \mathrm{pmolg}^{-1}$ less $B_{\max }$ than non-carriers of the $\mathrm{T}$ allele $(P=0.054)$. Among cases homozygous for the main $\mathrm{G}$ allele at rs2283265, those with the minor 5-repeat at intron8 have $41 \mathrm{pmolg}^{-1}$ less $B_{\max }$ than non-carriers of the intron8 5-repeat, on average ( $P=0.002$, Supplementary Table 6C).

For the rs6347 interaction model, among cases homozygous for the main A allele at rs6347, those with the minor $\mathrm{T}$ allele at rs2283265 have $28 \mathrm{pmolg}^{-1}$ less $B_{\max }$ than noncarriers of the T allele ( $P=0.09$, Supplementary Table 6D). Among cases homozygous for the main G allele at rs2283265, those with the minor $\mathrm{G}$ allele at rs6347 have $25 \mathrm{pmol} \mathrm{g}^{-1}$ less $B_{\max }$ than non-carriers of the $\mathrm{r} 6347 \mathrm{G}$ allele, on average $(P=0.024)$. It appears that the DRD2 rs2283265 interaction with either DAT variant is similar, with only a few subjects with discrepant genotypes.

To compare these results on protein activity with the outcomes ORs of the DAT-DRD2 interaction, we plotted the $D R D 2$ rs2283365 ORs for cocaine-related death along with the protein effects in Figure 1, grouped by DAT genotype, showing a reciprocal relationship. The relationship between $\mathrm{B}_{\max }\left(\mathrm{pmol} \mathrm{g}^{-1}\right)$ and DAT allele status changed significantly, and in opposite directions, depending on the rs2283265 genotype among case subjects, a mirror image of what is observed with cocaine-related risk ORs. The relationship between DAT haplotypes and DAT expression cannot be statistically analyzed, with $B_{\max }$ values for only threehaplotype 22 carrier subjects available.

\section{Discussion}

The results of this study reveal a profound interaction between regulatory polymorphisms in the genes encoding the D2 dopamine receptor and the dopamine transporter, in the context of cocaine abuse and death as a result of the abuse. These two key proteins physically bind to each other in the presynaptic terminal and are thought to modulate each other's functions. ${ }^{21-23,25}$ Expressed presynaptically, the D2S receptor splice isoform interacts with DAT and stabilizes it in the plasma membrane, ${ }^{21,22}$ so D2S levels influence the level of functional
DAT that is localized to the cell membrane. We now demonstrate that the lethal cocaine risk previously shown to be conferred by intronic DRD2 SNP rs2283265, which reduces D2S formation, ${ }^{1}$ is strongly enhanced from $\sim 3$ to $\sim 7$ in subjects homozygous for the major alleles of DAT variants rs3836790 (intron8 5/6-repeat) or rs6347 (exon9), even though these DAT variants had no significant effect when considered alone. In contrast, no significant risk was apparent for DRD2 rs2283265 in carriers of the DAT intron8 minor 5-repeat allele. We conclude that the 6repeat potentiates, whereas the 5-repeat blunts the impact of the $D R D 2$ rs2283265 risk allele. In a mirror image, the minor DAT intron8 5-repeat turned into a risk allele $(O R=2.1 ; P=0.02)$ (Table $3 b$ ) in homozygous carriers of the major DRD2 rs2283265 G allele. Conversely, the risk conveyed by a single minor allele in either one gene is counterbalanced by the presence of a minor allele in the other gene.

The role of interracial differences. The interactions between the $D A T$ and $D R D 2$ variants can account for our previous observation that the minor DRD2 rs2283265 allele did not seem to be a risk factor in African Americans. In this population, the rs2283265 risk allele has low frequency (7\%), whereas the protective intron8 5-repeat and the minor rs6347 allele are more abundant ( $\sim 66$ and $58 \%$, respectively). The model calculations suggest that race does not have a significant role if one accounts for these large allele frequency differences, suggesting that the DRD2/DAT interaction has similar effects regardless of genetic background. Although our results support the hypothesis that the DRD2 and DAT variants have similar effects regardless of race, a larger cohort is needed to address this question further.

Effects of DRD2-DAT variants on DAT protein activity. The elevated DAT protein activity, measured as ligand binding $B_{\text {max }}$, observed in the ventral putamen of cocaine abusers also appear to fall under the influence of interactions between DAT rs3836790 (rs6347 gave with similar results) and $D R D 2$ rs2283265. The minor DRD2 $\mathrm{T}$ allele of rs2283265 correlated with a significant reduction in DAT $B_{\max }$ in homozygous carriers of the main 6-repeat allele of $D A T$ intron8 rs3836790, whereas the 5-repeat allele correlated with a significant reduction in the context of the major DRD2 rs2283265 G allele (Figure 1b, Supplementary Table $6 \mathrm{C}$ ). The finding of a reciprocal genotype effect on DAT protein levels in cocaine abusers strengthens the hypothesis that these variants affect risk of cocaine abuse/ death. Therefore we need to consider what is known about these genetic variants at present, regarding regulation of gene expression. ${ }^{24,30}$

In $D R D 2$, we focused here only on splicing SNP rs2283265, which is in high LD with and therefore also represents rs1076560, whereas the promoter variant rs1076560 had insufficient allele frequency for analysis. Cocaine exposure did not appear to affect the influence of rs2283265 on splicing, arguing against a cocaine-genotype effect involving D2S and D2L formation. ${ }^{1}$ Nevertheless, a dynamic interaction with DAT at the protein level is feasible as rs2283265 reduces D2S formation, which binds to DAT presynaptically. ${ }^{24}$ With regards to $D A T$, the intron8 5-repeat mediates higher DAT minigene expression in vitro than the 6-repeat, displaying even higher 
expression when the cells are treated with cocaine. ${ }^{29,30}$ Similarly, the minor allele of rs6347 was also associated with increased expression in a cell culture model but it remains uncertain whether rs6347 has an independent effect in vivo. ${ }^{24}$ In human brain tissues, however, both the 5-repeat allele and the rs6347 minor allele are associated with lower DAT mRNA expression in human substantia nigra (without cocaine treatment). ${ }^{24}$ Previous work has shown that DAT protein activity, measured by DAT binding site densities and functional uptake, is upregulated by cocaine exposure, ${ }^{35,41-45}$ whereas mRNA levels remained constant in one study, ${ }^{45}$ suggesting regulation at the level of mRNA processing, translation and increased trafficking of DAT to the membrane to compensate for rapid shifts in dopamine in the synaptic cleft with cocaine abuse. These findings are not incompatible as the regulatory variants considered here operate at the level of RNA processing and possibly translation, so that overall mRNA levels and localized or distal DAT protein levels can differ substantially. Given the complex regulation of both DRD2 and DAT mRNA expression, and specifically processing and translation, further studies will be needed to reconcile earlier reports on DAT mRNA and protein levels under the influence of cocaine exposure.

The effect of DAT haplotype structure. DAT haplotypes could represent strong risk factors if two regulatory variants, the rs27072 SNP allele and intron8 5-repeat, are phased with each other on the same chromosome. DAT SNP rs27072 ( $3^{\prime}$ UTR, MAF 15\%), shown to be associated with bipolar disorder in one cohort, ${ }^{24}$ regulates DAT expression in vitro in a fashion that strongly depends on extent of expression-at low levels, the DAT mRNA carrying the minor allele appears to be sequestered, possibly by microRNAs, unable to support translation, which is partly overcome at high expression levels. ${ }^{24}$ Whether this dynamic mechanism has a role here cannot be tested as the effect size relative to MAF was insufficient for the study cohort to yield sufficient power. However, in the context of a haplotype with the intron8 5-repeat allele, it appears to represent a very strong risk factor for cocaine-related death. Owing to the increased allelic frequency of the 5-repeat in African Americans, there is greater opportunity for the haplotype consisting of the minor alleles of both the intron8 5/6-repeat and rs27072 (the 22 haplotype) to exist. We report here that the 22 haplotype occurred at a remarkable $\sim 19 \%$ frequency in African American former cocaine abusers ( $\sim$ one in 3 subjects being carriers). Odds ratios are difficult to determine as the control group 22 haplotype frequency was estimated at $0 \%$ in our cohort (with some ambiguity in haplotype assignment in compound heterozygotes), indicating strong risk conveyed by the 22 haplotype (Table 4). We hypothesize that the DAT 22 haplotype could be exquisitely sensitive to environmental stimuli, such as excessive cocaine exposure, adding a twovariant interaction in a single gene to the DAT-DRD2 genegene interaction model.

Possible mechanisms underlying the observed genegene-drug interactions. Several hypotheses might account for observed relationships between genotype, DAT protein levels and lethal cocaine exposure. One might speculate that upregulation of DAT activity caused by cocaine is a compensatory mechanism protecting against dopamine overload, whereas dampening DAT upregulation by the $D R D 2 / D A T$ risk allele combinations could enhance availability of dopamine upon cocaine exposure and hence result in excessive dopamine overload and toxicity. On the other hand, the DRD2-DAT risk allele combinations could also affect the risk of cocaine abuse, and progression to heavy abuse. It is noted that genotype effects on DAT protein activity were also detectable in non-cocaine abusers, albeit with lesser effect size. Furthermore, one needs to consider DAT-D2 effects in peripheral tissues that have a role in cocaine-related death. The process underlying cocainerelated death remains to be clarified; whether epistatic $D R D 2-D A T$ interactions occur in peripheral neurons remains to be investigated.

Scope and limitations of the study. This gene-gene-drug/ environment interaction study was performed on a small group of cases and matched controls; strong statistical significance was attained because of the robust epistatic interaction between $D R D 2$ and $D A T$ variants having demonstrable regulatory functions. Further studies will be needed to replicate these results and test under which other conditions this genetic interaction occurs. The study design further cannot distinguish between genetic effects on cocaine addiction, progression of abuse and death from cocaine exposure. Future studies must address the question whether the DRD2-DAT interactions also have a role in psychiatric disorders and in therapies with stimulant drugs or antipsychotics.

\section{Summary}

Given the evidence for interactions between DRD2 and $D A T{ }^{20}$ we assessed the interaction of regulatory variants in DRD2 and $D A T$, identifying a significant interaction between rs2283265 and DAT variant rs3836790 (intron8 5/6 repeat), and/or possibly rs6437. In light of the evidence for the effects of rs2283265 on D2S/L splicing and for the effects of rs3836790 on DAT expression, the interaction between these two variants in the context of cocaine abuse reported here is striking. As drug addiction is thought to be a complex, multigenic disorder involving a large number of genes with low penetrance, the finding of a significant interaction between two genetic variants in plausible candidate genes points to epistasis as a mechanism with the potential to close a gap in the 'missing heritability'.

\section{Conflict of interest}

The authors declare no conflict of interest.

Acknowledgements. This study was supported by the National Institutes of Health, U01 GM 092655 from the National Institute of General Medical Sciences (WS) and R01 DA022199 (WS), DA06227 (DCM) from the National Institute on Drug Abuse, and UL1RR025755 from the National Center For Research Resources.

1. Moyer RA, Wang D, Papp AC, Smith RM, Duque L, Mash DC et al. Intronic polymorphisms affecting alternative splicing of human dopamine D2 receptor are associated with cocaine abuse. Neuropsychopharmacology 2011; 36: 753-762. 
2. Manolio TA, Collins FS, Cox NJ, Goldstein DB, Hindorff LA, Hunter DJ et al. Finding the missing heritability of complex diseases. Nature 2009; 461: 747-753.

3. Zuk O, Hechter E, Sunyaev SR, Lander ES. The mystery of missing heritability: genetic interactions create phantom heritability. Proc Natl Acad Sci USA 2012; 109: 1193-1198.

4. Saiz PA, Garcia-Portilla MP, Arango C, Morales B, Arias B, Corcoran P et al. Genetic polymorphisms in the dopamine-2 receptor (DRD2), dopamine-3 receptor (DRD3), and dopamine transporter (SLC6A3) genes in schizophrenia: Data from an association study. Prog Neuropsychopharmacol Biol Psychiatry 2010; 34: 26-31.

5. Caldu X, Vendrell P, Bartres-Faz D, Clemente I, Bargallo N, Jurado MA et al. Impact of the COMT Val108/158 Met and DAT genotypes on prefrontal function in healthy subjects. Neuroimage 2007; 37: 1437-1444.

6. Yacubian J, Sommer T, Schroeder K, Glascher J, Kalisch R, Leuenberger B et al. Genegene interaction associated with neural reward sensitivity. Proc Natl Acad Sci USA 2007; 104: 8125-8130.

7. Tan HY, Chen AG, Chen Q, Browne LB, Verchinski B, Kolachana B et al. Epistatic interactions of AKT1 on human medial temporal lobe biology and pharmacogenetic implications. Mol Psychiatry 2012; 17: 1007-1016.

8. Nixon DC, Prust MJ, Sambataro F, Tan HY, Mattay VS, Weinberger DR et al. Interactive effects of DAOA (G72) and catechol-O-methyltransferase on neurophysiology in prefrontal cortex. Biol Psychiatry 2011; 69: 1006-1008.

9. Laucht M, Skowronek MH, Becker K, Schmidt MH, Esser G, Schulze TG et al. Interacting effects of the dopamine transporter gene and psychosocial adversity on attention-deficit hyperactivity disorder symptoms among 15-year-olds from a high-risk community sample. Arch Gen Psychiatry 2007; 64: 585-590.

10. Caspi A, Sugden K, Moffitt TE, Taylor A, Craig IW, Harrington $\mathrm{H}$ et al. Influence of life stress on depression: moderation by a polymorphism in the 5-HTT gene. Science 2003; 301: 386-389.

11. Bendesky A, Tsunozaki M, Rockman MV, Kruglyak L, Bargmann $\mathrm{Cl}$. Catecholamine receptor polymorphisms affect decision-making in C. elegans. Nature 2011; 472: 313-318.

12. Sadee W, Wang D, Papp AC, Pinsonneault JK, Smith RM, Moyer RA et al. Pharmacogenomics of the RNA world: structural RNA polymorphisms in drug therapy. Clin Pharmacol Ther 2011; 89: 355-365.

13. Wray GA. The evolutionary significance of cis-regulatory mutations. Nat Rev Genet 2007; 8: 206-216.

14. Epps $\mathrm{C}$, Holt $\mathrm{L}$. The genetic basis of addiction and relevant cellular mechanisms. Int Anesthesiol Clin 2011; 49: 3-14.

15. Sulzer D. How addictive drugs disrupt presynaptic dopamine neurotransmission. Neuron 2011; 69: 628-649.

16. Ciccarone D. Stimulant abuse: pharmacology, cocaine, methamphetamine, treatment, attempts at pharmacotherapy. Prim Care 2011; 38: 41-58.

17. Di Chiara G, Imperato A. Drugs abused by humans preferentially increase synaptic dopamine concentrations in the mesolimbic system of freely moving rats. Proc Natl Acad Sci USA 1988; 85: 5274-5278.

18. Usiello A, Baik JH, Rouge-Pont F, Picetti R, Dierich A, LeMeur M et al. Distinct functions of the two isoforms of dopamine D2 receptors. Nature 2000; 408: 199-203.

19. Khan ZU, Mrzljak L, Gutierrez A, de la Calle A, Goldman-Rakic PS. Prominence of the dopamine D2 short isoform in dopaminergic pathways. Proc Natl Acad Sci USA 1998; 95 $7731-7736$

20. Bertolino A, Fazio L, Di Giorgio A, Blasi G, Romano R, Taurisano P et al. Genetically determined interaction between the dopamine transporter and the D2 receptor on prefronto-striatal activity and volume in humans. J Neurosci 2009; 29: 1224-1234.

21. Lee FJ, Pei L, Moszczynska A, Vukusic B, Fletcher PJ, Liu F. Dopamine transporter cell surface localization facilitated by a direct interaction with the dopamine $\mathrm{D} 2$ receptor. $E M B O$ J 2007; 26: 2127-2136.

22. Bolan EA, Kivell B, Jaligam V, Oz M, Jayanthi LD, Han Y et al. D2 receptors regulate dopamine transporter function via an extracellular signal-regulated kinases 1 and 2 dependent and phosphoinositide 3 kinase-independent mechanism. Mol Pharmacol 2007; 71: $1222-1232$.

23. Jones SR, Gainetdinov RR, Hu XT, Cooper DC, Wightman RM, White FJ et al. Loss of autoreceptor functions in mice lacking the dopamine transporter. Nat Neurosci 1999; 2 : 649-655.

24. Pinsonneault JK, Han DD, Burdick KE, Kataki M, Bertolino A, Malhotra AK et al. Dopamine transporter gene variant affecting expression in human brain is associated with bipolar disorder. Neuropsychopharmacology 2011; 36: 1644-1655.

25. Blasi G, Lo Bianco L, Taurisano P, Gelao B, Romano R, Fazio L et al. Functional variation of the dopamine D2 receptor gene is associated with emotional control as well as brain activity and connectivity during emotion processing in humans. J Neurosci 2009; 29 : 14812-14819.
26. Brookes KJ, Neale BM, Sugden K, Khan N, Asherson P, D'Souza UM. Relationship between VNTR polymorphisms of the human dopamine transporter gene and expression in post-mortem midbrain tissue. Am J Med Genet B Neuropsychiatr Genet 2007; 144B 1070-1078.

27. Feng Y, Wigg KG, Makkar R, Ickowicz A, Pathare T, Tannock R et al. Sequence variation in the 3'-untranslated region of the dopamine transporter gene and attentiondeficit hyperactivity disorder (ADHD). Am J Med Genet B Neuropsychiatr Genet 2005; 139B: $1-6$.

28. Franke B, Vasquez AA, Johansson S, Hoogman M, Romanos J, Boreatti-Hummer A et al. Multicenter analysis of the SLC6A3/DAT1 VNTR haplotype in persistent ADHD suggests differential involvement of the gene in childhood and persistent ADHD. Neuropsychopharmacology 2010; 35: 656-664.

29. Guindalini C, Howard M, Haddley K, Laranjeira R, Collier D, Ammar N et al. A dopamine transporter gene functional variant associated with cocaine abuse in a Brazilian sample. Proc Natl Acad Sci USA 2006; 103: 4552-4557.

30. Hill M, Anney RJ, Gill M, Hawi Z. Functional analysis of intron 8 and $3^{\prime}$ UTR variable number of tandem repeats of SLC6A3: differential activity of intron 8 variants. Pharmacogenomics J 2010; 10: 442-447.

31. Preuss UW, Zill P, Koller G, Bondy B, Sokya M. D2 dopamine receptor gene haplotypes and their influence on alcohol and tobacco consumption magnitude in alcohol-dependent individuals. Alcohol Alcohol 2007; 42: 258-266.

32. Todd RD, Jong YJ, Lobos EA, Reich W, Heath AC, Neuman RJ. No association of the dopamine transporter gene 3' VNTR polymorphism with ADHD subtypes in a population sample of twins. Am J Med Genet 2001; 105: 745-748.

33. Zhang Y, Bertolino A, Fazio L, Blasi G, Rampino A, Romano R et al. Polymorphisms in human dopamine $\mathrm{D} 2$ receptor gene affect gene expression, splicing, and neuronal activity during working memory. Proc Natl Acad Sci USA 2007; 104: 20552-20557.

34. Kazantseva A, Gaysina D, Malykh S, Khusnutdinova E. The role of dopamine transporter (SLC6A3) and dopamine D2 receptor/ankyrin repeat and kinase domain containing 1 (DRD2/ANKK1) gene polymorphisms in personality traits. Prog Neuropsychopharmacol Biol Psychiatry 2011; 35: 1033-1040.

35. Mash DC, Pablo J, Ouyang Q, Hearn WL, Izenwasser S. Dopamine transport function is elevated in cocaine users. J Neurochem 2002; 81: 292-300.

36. Stephens BG, Jentzen JM, Karch S, Wetli CV, Mash DC. National Association of Medical Examiners position paper on the certification of cocaine-related deaths. Am J Forensic Med Pathol 2004; 25: 11-13.

37. Hosmer D, Lemeshow S. Applied Logistic Regression. 2nd edn. John Wiley \& Sons: New York, 2000.

38. Little RJA, Rubin DB. Statistical Analysis with Missing Data. 2nd edn. John Wiley \& Sons: New York, 2002.

39. Marchenko YV, Carroll RJ, Lin DY, Amos Cl, Gutierrez RG. Semiparametric analysis of case-control genetic data in the presence of environmental factors. Stata J 2008; 8 : 305-333.

40. Staley JK, Boja JW, Carroll FI, Seltzman HH, Wyrick CD, Lewin AH et al. Mapping dopamine transporters in the human brain with novel selective cocaine analog [125I]RTI121. Synapse 1995; 21: 364-372.

41. Staley JK, Hearn WL, Ruttenber AJ, Wetli CV, Mash DC. High affinity cocaine recognition sites on the dopamine transporter are elevated in fatal cocaine overdose victims. Pharmacol Exp Ther 1994; 271: 1678-1685

42. Little KY, Kirkman JA, Carroll FI, Clark TB, Duncan GE. Cocaine use increases [3H]WIN 35428 binding sites in human striatum. Brain Res 1993; 628: 17-25.

43. Little KY, McLaughlin DP, Zhang L, McFinton PR, Dalack GW, Cook EH Jr et al. Brain dopamine transporter messenger RNA and binding sites in cocaine users: a postmortem study. Arch Gen Psychiatry 1998; 55: 793-799.

44. Malison RT, Best SE, van Dyck CH, McCance EF, Wallace EA, Laruelle $M$ et al. Elevated striatal dopamine transporters during acute cocaine abstinence as measured by [123|] beta-CIT SPECT. Am J Psychiatry 1998; 155: 832-834.

45. Little KY, Elmer LW, Zhong H, Scheys JO, Zhang L. Cocaine induction of dopamine transporter trafficking to the plasma membrane. Mol Pharmacol 2002; 61: 436-445.
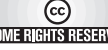

Translational Psychiatry is an open-access journal published by Nature Publishing Group. This work is licensed under the Creative Commons Attribution-NonCommercial-No Derivative Works 3.0 Unported License. To view a copy of this license, visit http://creativecommons.org/licenses/by-nc-nd/3.0/

Supplementary Information accompanies the paper on the Translational Psychiatry website (http://www.nature.com/tp) 